\title{
Avaliação toxicológica pré-clínica do chá das folhas de Morus nigra L. (Moraceae)
}

\author{
OLIVEIRA, A.C.B.'; OLIVEIRA, A.P.'; GUIMARÃES, A.L.'; OLIVEIRA, R.A.'; SILVA, F.S.'; REIS, S.A.G.B.'; \\ RIBEIRO, L.A.A. ${ }^{1}$; ALMEIDA, J.R.G.S. ${ }^{11}$ \\ ${ }^{1}$ Núcleo de Estudos e Pesquisas de Plantas Medicinais, Universidade Federal do Vale do São Francisco, 56.304- \\ 205, Petrolina, Pernambuco, Brasil. *jackson.guedes@univasf.edu.br
}

\begin{abstract}
RESUMO: O objetivo desse estudo foi realizar um ensaio toxicológico pré-clínico para analisar a toxicidade do chá das folhas de Morus nigra L. (Moraceae). A toxicidade subcrônica do chá (CF$\mathrm{Mn}$ ) foi avaliada durante 30 dias por via oral em ratos. Ao grupo controle foi administrado água, para comparação. Durante o período experimental foi avaliada a presença de sinais de toxicidade, variação do peso corporal, e o consumo de líquido e alimento. Ao final do experimento o sangue dos animais foi retirado para análise de parâmetros hematológicos e bioquímicos. Não foram observados mortalidade e sinais de toxicidade indicando baixa toxicidade da planta. Não houve alterações nos parâmetros hematológicos e bioquímicos. Nas condições do estudo, o CF-Mn pode ser considerado de baixa toxicidade, pois não produziu efeitos tóxicos nos animais tratados.
\end{abstract}

Palavras-chave: Toxicidade subcrônica, Morus nigra L., Moraceae

ABSTRACT: Pre-clinical toxicological evaluation of tea from the leaves of Morus nigra $L$. (Moraceae). The aim of this study was to carry out a pre-clinical toxicological assay to analyze the toxicity of tea from the leaves of Morus nigra L. (Moraceae). The subchronic toxicity of this tea (CF-Mn) was orally evaluated during 30 days in rats. The control group was given water for comparison. During the experimental period, signs of toxicity, body weight variation, and water and food consumption were assessed. At the end of the experiment, the blood of animals was removed for analysis of hematological and biochemical parameters. No mortality and no toxicity signs were observed, indicating low toxicity of the plant. There was no alteration in the hematological and biochemical parameters. Under the study conditions, CF-Mn can be considered of low toxicity since it did not produce toxic effects in treated animals.

Keywords: Subchronic toxicity, Morus nigra L., Moraceae

\section{INTRODUÇÃO}

Morus nigra L. é uma espécie pertencente ao gênero Morus, família Moraceae. Esse gênero possui cerca de 24 espécies e uma subespécie, com pelo menos 100 variedades conhecidas. A planta pode ser encontrada em regiões temperadas e subtropicais e pode crescer em uma grande variedade de condições climáticas, topográficas e de solo (Ercisli \& Orhan, 2007).

Na região do Vale do São Francisco Brasil, essa espécie é conhecida popularmente como "amora-miúra". O chá das folhas (decocto) é bastante utilizado pela população local para o tratamento de diabetes, colesterol, problemas cardiovasculares, obesidade e gota. A planta foi trazida para a região por imigrantes japoneses, adaptando-se bem às condições de clima e solo.

Algumas espécies do gênero Morus são amplamente cultivadas na China e no Japão porque suas folhas servem como alimento para o bicho-daseda (Nomura, 1988). Plantas desse gênero também têm sido usadas na medicina tradicional chinesa como antiflogístico, hepatoprotetor, hipotensor, antipirético, analgésico, diurético, expectorante, antidiabético (Nomura, 1988; Chen et al., 1995) bem como para o tratamento de anemia e artrite (Ozgen et al., 2009). Um alcaloide piperidínico (moranolina) e glicoproteínas foram isolados como agentes antidiabéticos das raízes e folhas de diversas espécies de Morus (Kim et al., 1999). As folhas dessas espécies são consumidas no Japão e Coréia como agentes antihiperglicêmicos por pacientes com diabetes mellitus, devido à presença da 1-desoxinojirimicina, que é conhecida como um dos mais potentes inibidores da á-glicosidase (Kim

Recebido para publicação em 26/05/2011

Aceito para publicação em 22/11/2012

Rev. Bras. PI. Med., Campinas, v.15, n.2, p.244-249, 2013. 
et al., 2003).

Este gênero contém uma variedade de compostos fenólicos, incluindo flavonoides isoprenilados, estilbenos, 2-arilbenzopiranos, cumarinas, cromonas, xantonas e uma variedade de compostos de Diels-Alder (Nomura et al., 1988; Nomura \& Hano, 1994). Alguns desses compostos exibem propriedades biológicas interessantes tais como efeito antiflogístico, antiinflamatório, diurético, hipotensor, e alguns são também conhecidos como fitoalexinas (Syah et al., 2000). O potencial antioxidante de alguns compostos fenólicos de Morus alba também foi reportado na literatura (Fukai et al., 2003).

Há poucos estudos envolvendo a composição química e a avaliação das propriedades biológicas e farmacológicas de Morus nigra. Morusina, um prenilflavonoide isolado das raízes dessa espécie mostrou propriedades antinociceptivas em modelos de dor em camundongos (Souza et al., 2000). Em estudo recente, Naderi et al. (2004) demonstrou que os extratos dos frutos de Morus nigra têm uma ação protetora contra o dano peroxidativo de biomembranas e biomoléculas. Dois novos flavonoides prenilados, momigrol $\mathrm{E}$ e momigrol $\mathrm{F}$ foram isolados das cascas do caule dessa espécie (Wang et al., 2009), bem como ácido betulínico, â-sitosterol e germanicol (Padilha et al., 2010). Propriedades antinociceptiva e antiinflamatória também foram reportadas para o extrato diclorometano das folhas da planta (Padilha et al., 2009; Padilha et al., 2010). Recentemente, nosso grupo de pesquisa avaliou o potencial hipoglicemiante e a toxicologia do extrato etanólico bruto dessa espécie (Almeida et al., 2011).

Tendo em vista o extenso uso popular do chá das folhas de Morus nigra na nossa região e a escassez de estudos sobre a toxicologia dessa espécie, o objetivo do presente estudo foi avaliar a toxicidade subcrônica do chá das folhas, preparado de acordo com o seu uso popular, visando avaliar parâmetros de segurança de uso bem como alterações no peso, consumo de líquido e consumo de alimento dos animais.

\section{MATERIAL E MÉTODOS}

\section{Material botânico}

As folhas de Morus nigra $L$. foram coletadas na Fazenda Ouro Verde, município de Casa NovaBA em fevereiro de 2010. Uma exsicata da planta (1764) está depositada no Herbário da Universidade Federal do Vale do São Francisco (HVASF).

\section{Preparação do chá das folhas de Morus nigra L. (CF-Mn)}

A escolha da parte da planta e do tipo de extração a ser utilizada foi baseada nas informações de uso popular, pois a planta é consumida na forma de chá (decocto). O chá das folhas foi preparado diariamente da mesma maneira como é preparado pela população. As folhas foram secas à sombra e depois foram trituradas manualmente. Em seguida, o pó $(12 \mathrm{~g})$ foi submetido a uma extração por decocção em água $(1000 \mathrm{~mL})$ a $100^{\circ} \mathrm{C}$ durante 10 minutos. Após o resfriamento do material, o decocto foi administrado diariamente aos animais.

\section{Animais}

Foram utilizados ratos Wistar machos (Rattus novergicus), pesando entre 250-300 g, provenientes do Biotério da Universidade Federal do Vale do São Francisco (UNIVASF). Todos os animais foram mantidos sob condições controladas de temperatura $\left(22 \pm 2^{\circ} \mathrm{C}\right)$ com livre acesso à alimentação e água, ciclo claro-escuro de 12 horas, com a fase de luz iniciando às 06:00 e terminando às 18:00 horas. Todos os protocolos experimentais foram aprovados pelo Comitê de Ética em Pesquisa da Universidade Federal do Vale do São Francisco sob o número 22021098.

\section{Avaliação da toxicidade subcrônica}

Os ratos $(n=20)$ foram divididos em 4 grupos de cinco animais cada. Cada grupo foi submetido a condições controladas de fornecimento de líquido e alimento durante 30 dias consecutivos. Os grupos I e II foram utilizados como controle e os grupos III e IV como grupos experimentais. Os dois primeiros grupos receberam diariamente 500 $\mathrm{mL}$ de água potável em frascos de polipropileno e $200 \mathrm{~g}$ de ração, enquanto os outros dois grupos receberam $500 \mathrm{~mL}$ do chá das folhas e $200 \mathrm{~g}$ de ração. O peso dos animais, o consumo de líquido e de alimentos foi medido diariamente durante os 30 dias do experimento. Durante o período experimental, todos os animais foram observados para a verificação de algum sinal de toxicidade. A glicemia foi medida a cada 7 dias, durante 28 dias do experimento. Os valores da glicemia foram obtidos através de fitas reagentes (Accu-Chek® Active, Roche) acopladas a um glicosímetro digital portátil a partir de amostras de sangue coletadas da cauda do animal. Após o período experimental, os animais foram anestesiados com tiopental sódico e tiveram o sangue coletado para a avaliação de parâmetros hematológicos e bioquímicos.

\section{Análise de parâmetros hematológicos e bioquímicos do sangue \\ Para a avaliação de parâmetros} hematológicos e bioquímicos do sangue dos animais, foi utilizada a metodologia descrita por Vasconcelos et al. (2007) com modificações. Foi

Rev. Bras. PI. Med., Campinas, v.15, n.2, p.244-249, 2013. 
retirado sangue dos animais através do plexo braquial para análise de parâmetros hematológicos como: número de hemácias $\left(10^{6} / \mathrm{mm}^{3}\right)$, hemoglobina ( $\mathrm{g} / \mathrm{dL})$, hematócrito (\%), volume corpuscular médio $\left(\mathrm{VCM}, \mu^{3}\right)$, hemoglobina corpuscular média (HCM, $\mu \mathrm{g})$, concentração de hemoglobina corpuscular média $(\mathrm{CHCM}, \%)$, leucócitos $\left(10^{3} / \mathrm{mm}^{3}\right)$, neutrófilos bastonetes $(\%)$, neutrófilos segmentados (\%), eosinófilos (\%), linfócitos típicos (\%), monócitos (\%) e plaquetas $\left(10^{3} / \mathrm{mm}^{3}\right)$. Os parâmetros bioquímicos analisados em amostras de soro foram: glicose $(\mathrm{mg} / \mathrm{dL})$, colesterol $(\mathrm{mg} / \mathrm{dL})$, triglicerídeos $(\mathrm{mg} / \mathrm{dL})$, AST/TGO (U/L), ALT/TGP (U/L), uréia $(\mathrm{mg} / \mathrm{dL})$, ácido úrico $(\mathrm{mg} / \mathrm{dL})$, creatinina $(\mathrm{mg} / \mathrm{dL})$, albumina $(\mathrm{g} / \mathrm{dL})$ e amilase (U/L). Para a determinação dos parâmetros hematológicos foi utilizado um analisador hematológico Sysmex XT-2000, para os parâmetros bioquímicos utilizou-se um analisador automático Wiener BT 3000 Plus.

\section{Análise estatística}

Os resultados foram expressos como média \pm erro padrão da média considerando-se o número de 10 animais para os grupos controle e experimental. O nível de significância estatística foi determinado usando o Teste $t$ de Student. Os valores foram considerados estatisticamente significantes quando $p<0,05$.

\section{RESULTADOS E DISCUSSÃO}

O uso de plantas medicinais tem sido muito significativo em diversas populações, no entanto, o uso popular, e mesmo o tradicional, não são suficientes para validar eticamente as plantas medicinais como medicamentos eficazes e seguros. Faz-se necessário a realização de estudos toxicológicos para a avaliação de parâmetros de segurança, os quais não são observados pela utilização popular dessas plantas.

A população consome grande quantidade do chá das folhas de Morus nigra na região do Vale do São Francisco, chegando a substituir a ingestão diária de água pela ingestão do chá. A fitoterapia recomenda um consumo de 3 a 4 doses diárias de um chá, para que o tratamento possa ser efetivo e sem efeitos tóxicos (Brasil, 2011). Assim, nosso objetivo foi avaliar se a ingestão diária do chá, em substituição à água, poderia provocar efeitos tóxicos ou mesmo a morte dos animais. No ensaio toxicológico pré-clínico subcrônico para avaliação do efeito tóxico do chá das folhas de Morus nigra L. (CF-Mn), não foi observado morte nem sinais de toxicidade em nenhum dos grupos que receberam o chá diariamente durante os 30 dias do experimento. Como não houve mortes, podemos considerar o CF-Mn de baixa toxicidade.
Os animais tratados não mostraram qualquer alteração significante em relação ao peso corporal (Tabela 1). Geralmente, as alterações no peso corporal de animais tratados com substâncias refletem seus efeitos tóxicos, e têm sido utilizadas como indicadores de efeitos adversos de medicamentos e produtos químicos, especialmente se tal perda for maior do que 10\% do peso inicial (Subramanion, 2011). Foi observada diferença estatística em relação ao consumo de líquido nos primeiros 15 dias, pois houve uma diminuição do consumo no grupo experimental em relação ao controle. O consumo ficou normal nos últimos 15 dias, com média de 754,00 $\pm 37,17 \mathrm{~mL}$ para o grupo controle, e de $684,30 \pm 29,47 \mathrm{~mL}$ para o grupo experimental (Tabela 2), mas nenhuma diferença foi observada em relação ao consumo de alimentos (Tabela 3).

A análise da ingestão de alimentos e água em uma experimentação animal é importante para investigar a segurança das substâncias estudadas com intuito terapêutico (Mukinda; Eagles, 2010). Como não houve diferença no ganho de peso nem no consumo de alimentos, podemos considerar o chá de baixa toxicidade.

Todos os resultados apresentados nas tabelas representam as médias dos valores obtidos nos primeiros e nos últimos 15 dias, bem como a média dos valores após o período de 30 dias.

Um dos principais usos populares do chá da planta na nossa região é para o tratamento do diabetes mellitus, o que tem sido uma prática constante por parte da população. Existe uma

TABELA 1. Média do ganho de peso de ratos tratados com o chá das folhas de Morus nigra durante 30 dias $(n=10)$.

\begin{tabular}{ccc}
\hline Tempo(dias) & \multicolumn{2}{c}{ Peso (g) } \\
\cline { 2 - 3 } & Controle & Experimental \\
\hline $1-15$ & $303,10 \pm 1,96$ & $302,50 \pm 1,81$ \\
$16-30$ & $322,10 \pm 1,68$ & $325,7 \pm 1,77$ \\
30 & $312,60 \pm 2,17$ & $314,10 \pm 2,49$ \\
\hline
\end{tabular}

TABELA 2. Média do consumo de líquido de ratos tratados com o chá das folhas de Morus nigra durante 30 dias $(n=10)$.

\begin{tabular}{ccc}
\hline Tempo(dias) & \multicolumn{2}{c}{ Consumo de líquido $(\mathrm{mL})$} \\
\cline { 2 - 3 } & Controle & Experimental \\
\hline $1-15$ & $712,00 \pm 17,39$ & $546,00 \pm 25,17^{* * *}$ \\
$16-30$ & $754,00 \pm 37,17$ & $684,30 \pm 29,47$ \\
30 & $733,00 \pm 20,54$ & $615,20 \pm 22,97^{* * *}$ \\
\hline${ }^{* * *} p<0,0001$. & Teste t de Student a $5 \%$ de probabilidade.
\end{tabular}


TABELA 3. Média do consumo de alimento de ratos tratados com o chá das folhas de Morus nigra durante 30 dias $(n=10)$.

\begin{tabular}{ccc}
\hline Tempo(dias) & \multicolumn{2}{c}{ Consumo de alimento (g) } \\
\cline { 2 - 3 } & Controle & Experimental \\
\hline $1-15$ & $265,40 \pm 4,82$ & $254,50 \pm 6,02$ \\
$16-30$ & $234,30 \pm 7,21$ & $227,90 \pm 7,84$ \\
30 & $249,90 \pm 5,15$ & $241,20 \pm 5,45$ \\
\hline
\end{tabular}

grande preocupação quanto ao uso seguro das plantas medicinais, principalmente em relação àquelas usadas para o tratamento de doenças crônicas, como o diabetes, pois muitos pacientes abandonam o tratamento convencional à base de insulina e fármacos hipoglicemiantes orais para utilizar exclusivamente o chá. Faz-se necessário não só estudos farmacológicos para avaliação do potencial terapêutico das plantas como também a realização de estudos toxicológicos pré-clínicos e clínicos. Assim, decidimos investigar ao longo do experimento os efeitos do chá sobre a glicemia em ratos normoglicêmicos. Os valores da glicemia foram obtidos a cada 7 dias através de fitas reagentes a partir de amostras de sangue coletadas da cauda do animal. Conforme mostrado na Tabela 4, não houve diferença estatística dos valores de glicemia obtidos dos animais experimentais em relação ao grupo controle, demonstrando que a planta não produziu o efeito hipoglicemiante esperado. Estudos mostram que os extratos de espécies do gênero Morus são efetivos agentes hipoglicemiantes. É possível sugerir que o efeito farmacológico esperado pode ser atribuído a uma situação crônica e a sintomas hiperglicêmicos. Estudos posteriores serão realizados através da indução de diabetes experimental com aloxana ou estreptozotocina para avaliação da atividade hipoglicemiante.

De maneira geral, não foi observada variação significativa nos parâmetros hematológicos dos grupos tratados com CF-Mn em relação ao controle (Tabela 5). Entretanto, um aumento no número de monócitos e uma diminuição do número de plaquetas foram observados no grupo experimental quando comparado com o controle. Essas alterações serão investigadas de maneira mais detalhada.

TABELA 4. Valores de glicemia obtidos após a administração do chá de Morus nigra durante 28 dias ( $n=10)$.

\begin{tabular}{lcccc}
\hline Grupos & \multicolumn{4}{c}{ Valores de glicemia } \\
\cline { 2 - 5 } & Basal & Após 7 dias & Após 14 dias & Após 28 dias \\
\hline Controle & $86,56 \pm 2,35$ & $78,25 \pm 1,75$ & $78,00 \pm 3,42$ & $66,67 \pm 2,36$ \\
Experimental & $83,30 \pm 2,74$ & $80,25 \pm 1,33$ & $80,71 \pm 2,91$ & $71,30 \pm 2,40$ \\
\hline
\end{tabular}

TABELA 5. Parâmetros hematológicos obtidos do sangue total de ratos tratados com o chá das folhas de Morus nigra durante 30 dias (toxicidade subcrônica).

\begin{tabular}{lll}
\hline Parâmetros & \multicolumn{2}{c}{ Tratamento } \\
\cline { 2 - 3 } & Controle & Experimental \\
\hline Hemácias $\left(10^{6} / \mathrm{mm}^{3}\right)$ & $9,28 \pm 0,32$ & $9,02 \pm 0,19$ \\
Hemoglobina $(\mathrm{g} / \mathrm{dL})$ & $16,37 \pm 0,68$ & $15,71 \pm 0,33$ \\
Hematócrito $(\%)$ & $51,83 \pm 1,98$ & $49,92 \pm 1,18$ \\
VCM $\left(\mu^{3}\right)$ & $55,82 \pm 0,65$ & $54,79 \pm 0,71$ \\
HCM $(\mu \mathrm{g})$ & $17,60 \pm 0,24$ & $17,45 \pm 0,18$ \\
CHCM $(\%)$ & $31,54 \pm 0,13$ & $31,59 \pm 0,13$ \\
Leucócitos $\left(10^{3} / \mathrm{mm}^{3}\right)$ & $3,40 \pm 0,34$ & $4,27 \pm 0,30$ \\
Neutrófilos bastonetes $(\%)$ & $0,40 \pm 0,22$ & $0,10 \pm 0,10$ \\
Neutrófilos segmentados $(\%)$ & $27,80 \pm 2,22$ & $26,40 \pm 1,25$ \\
Eosinófilos $(\%)$ & $0,50 \pm 0,27$ & $0,60 \pm 0,22$ \\
Linfócitos típicos $(\%)$ & $69,30 \pm 2,04$ & $68,80 \pm 1,38$ \\
Monócitos $(\%)$ & $2,20 \pm 0,25$ & $4,10 \pm 0,50^{* *}$ \\
Plaquetas $\left(10^{3} / \mathrm{mm}^{3}\right)$ & $741,9 \pm 36,45$ & $729,5 \pm 18,46^{* *}$ \\
\hline
\end{tabular}

Os valores estão expressos como média \pm e.p.m, $\mathrm{n}=10$ animais. Teste $t$ de Student a $5 \%$ de probabilidade. ${ }^{* *} \mathrm{p}<0,05$.

Rev. Bras. PI. Med., Campinas, v.15, n.2, p.244-249, 2013. 
TABELA 6. Parâmetros bioquímicos obtidos do soro de ratos tratados com o chá das folhas de Morus nigra durante 30 dias (toxicidade subcrônica).

\begin{tabular}{lll}
\hline Parâmetros & Tratamento & \\
\cline { 2 - 3 } & Controle & Experimental \\
\hline Glicose $(\mathrm{mg} / \mathrm{dL})$ & $66,67 \pm 2,36$ & $71,30 \pm 2,40$ \\
Colesterol (mg/dL) & $48,50 \pm 3,16$ & $46,50 \pm 3,73$ \\
Triglicerídeos (mg/dL) & $57,50 \pm 6,28$ & $65,33 \pm 3,93$ \\
AST/TGO (U/L) & $167,40 \pm 6,64$ & $158,90 \pm 6,63$ \\
ALT/TGP (U/L) & $62,10 \pm 2,76$ & $63,90 \pm 4,35$ \\
Uréia (mg/dL) & $59,50 \pm 2,81$ & $59,80 \pm 2,39$ \\
Ácido úrico (mg/dL) & $4,20 \pm 0,41$ & $4,23 \pm 0,45$ \\
Creatinina (mg/dL) & $1,03 \pm 0,03$ & $1,06 \pm 0,03$ \\
Albumina (g/dL) & $4,06 \pm 0,09$ & $4,08 \pm 0,08$ \\
Amilase (U/L) & $587,80 \pm 20,86$ & $590,90 \pm 14,80$ \\
\hline Os valores estão expressos como média te.p.m. $\mathrm{n}=10$ animais. Teste $t$ de Student a $5 \%$ de probabilidade.
\end{tabular}

Os resultados bioquímicos também não deram significância estatística em relação ao grupo controle (Tabela 6).

Apenas um estudo (Hemmati et al., 2008) havia sido citado na literatura com o objetivo de investigar os efeitos do extrato aquoso das folhas de Morus nigra sobre o fígado e rim de camundongos diabéticos. Nesse estudo, o diabetes foi induzido com estreptozotocina e o extrato das folhas foi administrado por via oral na dose de $1 \mathrm{mg} / \mathrm{kg}$ duas vezes ao dia, durante 42 dias. O extrato causou um aumento dos níveis séricos de creatinina, TGO e TGP nos animais. Em estudo recente realizado pelo nosso grupo (Almeida et al., 2011), avaliamos o potencial hipoglicemiante e a toxicidade aguda do extrato etanólico bruto das folhas de Morus nigra, e pudemos observar que o extrato pode ser considerado de baixa toxicidade, visto que não provocou morte nem a alteração de parâmetros bioquímicos e hematológicos nos animais após a administração de doses de $5 \mathrm{~g} / \mathrm{kg}$ e $2 \mathrm{~g} / \mathrm{kg}$, por via oral e intraperitoneal, respectivamente.

\section{CONCLUSÃO}

Com base nos resultados apresentados nesse estudo, podemos concluir que o chá das folhas de Morus nigra pode ser considerado de baixa toxicidade, pois a administração subcrônica durante o período de 30 dias por via oral não produziu mortes nem sinais de toxicidade nos animais. Além disso, não provocou alteração nos parâmetros bioquímicos e hematológicos analisados. Os órgãos dos animais (fígado, rins, coração e pulmões) foram retirados e serão encaminhados para análise histopatológica para a verificação de alterações microscópicas nas células. A análise macroscópica não revelou alterações nos órgãos. Com esses estudos de toxicidade podemos ter a noção de parâmetros de segurança, o que nos possibilita avançar para a realização de estudos clínicos, a fim de comprovar os benefícios terapêuticos da planta.

\section{AGRADECIMENTOS}

Os autores agradecem ao Conselho Nacional de Desenvolvimento Científico e Tecnológico (CNPq) e à Fundação de Amparo à Ciência e Tecnologia do Estado de Pernambuco (FACEPE) pelo auxílio financeiro.

\section{REFERÊNCIA}

ALMEIDA, J.R.G.S.; GUIMARÃES, A.L.; OLIVEIRA, A.P.; ARAÚJO, E.C.C.; SILVA, F.S.; NEVES, L.F.; OLIVEIRA, R.A.; SÁ, P.G.S.; QUINTANS-JÚNIOR, L.J. Evaluation of hypoglycemic potential and pre-clinical toxicology of Morus nigra L. (Moraceae). Latin American Journal of Pharmacy, v.30, p.96-100, 2011.

BRASIL. Agência Nacional de Vigilância Sanitária. Formulário de Fitoterápicos da Farmacopéia Brasileira / Agência Nacional de Vigilância Sanitária. Brasília: Anvisa, 2011, 126p.

CHEN, F.J.; NAKASHIMA, N.; KIMURA, I.; KIMURA, M. Hypoglicemic activity and mechanisms of extracts from mulberry leaves (folium mori) and cortex mori radicis in streptozotocin-induced diabetic mice. Yakugaku Zasshi, v.115, p.476-482, 1995.

ERCISLI, S.; ORHAN, E. Chemical composition of white (Morus alba), red (Morus rubra) and black (Morus nigra) mulberry fruits. Food Chemistry, v.103, p.1380-1384, 2007.

FUKAI, T.; SATOH, K.; NOMURA, T.; SAKAGAMI, H. Antinephritis and radical scavenging activities of prenylflavonoids. Fitoterapia, v.74, p.720-724, 2003.

Rev. Bras. PI. Med., Campinas, v.15, n.2, p.244-249, 2013. 
HEMMATI, A.A.; JALAI, M.H.; RASSHIDI, I.; HORMOZI, T.K. Effects of aqueous extract of black mulberry (Morus nigra) on liver and kidney of diabetic mice. Toxicology Letters, v.180, p.S48-S49, 2008.

KIM, E.S.; PARK, S.J.; LEE, E.J.; KIM, B.K.; HUH, H.; LEE, B.J. Purification and characterization of Moran 20K from Morus alba. Archives of Pharmaceutical Research, v.22, p.9-12, 1999.

KIM, J.W.; KIM, S.U.; LEE, H.S.; KIM, I.; AHN, M.Y.; RYU, K.S. Determination of 1-deoxynojirimycin in Morus alba L. leaves by derivatization with 9-fluorenylmethyl chloroformate followed by reversed-phase highperformance liquid chromatography. Journal of Chromatography A, v.1002, p.93-99, 2003.

MUKINDA, J.T.; EAGLES, P.F.K. Acute and subchronic oral toxicity profiles of the aqueous extract of Polygala fruticosa in female mice and rats. Journal of Ethnopharmacology, v. 128, p. 236-240, 2010.

NADERI, G.A.; ASGARY, S.; SARRAF-ZADEGAN, N.; OROOJY, H.; AFSHIN-NYA, F. Antioxidant activity of three extracts of Morus nigra. Phytotherapy Research, v.18, p.365-369, 2004.

NOMURA, T. Phenolic compounds of the mulberry tree and related plants. Progress in the Chemistry of Organic Natural Products, v. 53, p. 87-201, 1988.

NOMURA, T.; HANO, Y. Isoprenoid-substituted phenolic compounds of moraceous plants. Natural Products Reports, v.11, p.205-218, 1994.

OZGEN, M.; SERÇE, S.; KAYA, C. Phytochemical and antioxidant properties of anthocyanin-rich Morus nigra and Morus rubra fruits. Scientia Horticulturae, v.119, p.275-279, 2009.
PADILHA, M.M.; VILELA, F.C.; SILVA, M.J.D.; SANTOS, M.H.; ALVES-DA-SILVA, G.; GIUSTI-PAIVA, A. Antinociceptive effect of Morus nigra leaves in mice. Journal of Medicinal Food, v.12, n.6, p.1381-1385, 2009.

PADILHA, M.M.; VILELA, F.C.; ROCHA, C.Q.; DIAS, M.J.; SONCINI, R.; SANTOS, M.H.; ALVES-DA-SILVA, G.; GIUSTI-PAIVA, A. Antiinflammatory properties of Morus nigra leaves. Phytotherapy Research, 2010. DOI: $10.1002 /$ ptr.3134.

SOUZA, M.M.; BITTAR, M.; CECHINEL-FILHO, V.; YUNES, R.A.; MESSANA, I.; DELLE-MONACHE, F.; FERRARI, F. Antinociceptive properties of morusin, a prenylflavonoid isolated from Morus nigra root bark. Zeitschrift für Naturforschung, v. 55c, p. 256-260, 2000.

SUBRAMANION, L.J., ZURAINI, Z.; YENG, C.; YEE, L.L.; LACHIMANAN, Y.L.; SREENIVASAN, S. Acute oral toxicity of methanolic seed extract of Cassia fistula in mice. Molecules, v. 16, p. 5268-5282, 2011.

SYAH, Y.M.; ACHMAD, S.A.; GHISALBERTI, E.L.; HAKIM, E.H.; IMAN, M.Z.N.; MAKMUR, L.; MUJAHIDDIN, D. Andalasin A, a new stilbene dimmer from Morus macroura. Fitoterapia, v.71, p.630-635, 2000.

VASCONCELOS, T.H.C.; MODESTO-FILHO, J.; DINIZ, M.F.F.M.; SANTOS, H.B.; AGUIAR, F.B.; MOREIRA, P.V.L. Estudo toxicológico pré-clínico agudo com o extrato hidroalcoólco das folhas de Cissus sicyoides L. (Vitaceae). Brazilian Journal of Pharmacognosy, v.17, p.583-591, 2007.

WANG, L.; GONG, T.; CHEN, R.Y. Two new prenylflavonoids from Morus nigra L. Chinese Chemical Letters, v.20, p.1469-1471, 2009. 